\title{
The Evaluation of Parameter $M$ in the Big M Method of Linear Programming
}

\author{
Song Zhenzhen \\ Yinchuan Energy institute, Yinchuan, Ningxia, China \\ zhenzhen@163.com
}

Keywords: linear programming; simplex method; big M method; parameter evaluation

\begin{abstract}
In the simplex method of linear programming, there is a big M method (the penalty factor method)forfinding an initial feasible basis. The current textbooks of operationsresearch only explain that the big $\mathrm{M}$ method isefficient when $\mathrm{M}$ is large enough, and never give precise evaluation to the parameter $\mathrm{M}$. This paper determines a constant $\mathrm{M}_{0}$ and proves that the big $\mathrm{M}$ method is convergent to an optimal solution of the primal problem when $\mathrm{M}>\mathrm{M}_{0}$.
\end{abstract}

\section{Introduction}

Linear programming is the basic part of the operations research. Simplex method for solving linear programming problems, you must first determine an initial basic feasible solution in no obvious initial basic feasible solution, usually by means of artificial variables to construct an initial feasible basis, namely in the standard linear programming (linear programming standard mentioned in this paper are the target constraints in the minimization model) the introduction of nonnegative artificial variables, construct a unit matrix, and then change the objective function of the original, with two stage method or $M$ method to solve the two stage method, the objective function is established for the initial basic feasible solution of the original problem. The advantages of the big $\mathrm{M}$ method in $\mathrm{M}$ to choose the right (large enough), cannot in stages but choose to calculate how big $\mathrm{M}$, generally speaking, there is no formula can be moved by a big $\mathrm{M}$ method is that $\mathrm{M}$ has always been involved in the iterative calculation process is very complicated; and the two stage method is the objective function of the two stages are not consistent.

The normal form is:

$$
\begin{gathered}
\min \sum_{j=1}^{n} c_{j} x_{j} \\
\left\{\begin{array}{l}
\sum_{j=1}^{n} a_{i j} x_{j}=b_{j}(i=1,2, \ldots m) \\
x_{j} \geq 0(j=1,2, \ldots n)
\end{array}\right.
\end{gathered}
$$

We transform it to:

$$
\begin{gathered}
\min \sum_{j=1}^{n} c_{j} x_{j}+M \sum_{i=1}^{m} x_{n+i} \\
\left\{\begin{array}{l}
\sum_{j=1}^{n} a_{i j} x_{j}+x_{n+i}=b_{i}(i=1,2, \ldots m) \\
x_{j} \geq 0(j=1,2, \ldots n+m)
\end{array}\right.
\end{gathered}
$$

The shortcomings of the big $M$ method are: value is not easy to determine the $M$ and $M$ easily lead to the calculation error. Thus, $m$ the valuation problem is indeed the key of this method. Two stage methods emerges as the times require, it is the force to minimum as the first stage, the 
minimum value $\Delta=\sum_{i=1}^{m} x_{n+i}$ as the second stage, quite to deal with two different linear programming. The parameter $\mathrm{M}$ of valuation, but with increased model complexity cost (iterative process every step of the calculation table to with two lines to record two objective function test.

\section{Natures of Feasible Solution}

\section{Basic Form}

According to custom, the standard form of linear programming can be abbreviated as

$$
\min c^{T} x ; A x=b, x \geq 0
$$

We extend it to:

$$
\min \left(c^{T}, M e^{T}\right)\left(\begin{array}{l}
x \\
y
\end{array}\right) ;(A, I)\left(\begin{array}{l}
x \\
y
\end{array}\right)=b\left(\begin{array}{l}
x \\
y
\end{array}\right) \geq 0
$$

In this formula, $e^{T}=(1,1, \cdots 1)$ is an $\mathrm{m}$ dimensional vector corresponding variables in the $\mathrm{B}$ column of said base variables; all base variables constitute an m-dimensional vector for $\mathrm{X}_{\mathrm{B}}$. Corresponding to variables outside the column of $\mathrm{B}$ said for non-basic variables; all variables constitute the $n$ dimensional vector remember $X_{N}$, corresponding to base $B$ solution $\left(X_{B}, X_{N}\right)$ by the following equations uniquely determined.

$$
B x_{B}=b, x_{N}=0
$$

Because the former is $m$ variable $m$ equations of linear equation group, and the coefficient matrix $\mathrm{B}$ is nonsingular, the rule was the only solution. Notable is, base solution is constraint equation solution, not necessarily satisfy the negative constraints. If $X_{B}$ is more than or equal to 0 , the basic solution $\left(\mathrm{X}_{\mathrm{B}}, \mathrm{X}_{\mathrm{N}}\right)$ is a linear programming solution, so that corresponding to the base $\mathrm{B}$ basic feasible solution. At this point, the base $\mathrm{B}$ is called feasible base of linear programming basic theorem: if a linear programming optimal solution, in a basic feasible solution is reached. The simplex method is to construct a basic feasible solution of the sequence.The objective function values gradually decreased, and finally achieve the optimal basic feasible solution.

\section{Basic Natures}

The process ofan upper bound estimate of the value of the base variables is improved, and a lower bound estimate is added to the following lemma 1.

$$
|\operatorname{det} B| \leq\left\|b_{1}\right\| \ldots\left\|b_{m}\right\|
$$

Lemma 1: If $\alpha=\max \left\{\left|a_{i j}\right|: 1 \leq i \leq m, 1 \leq j \leq n\right\}, \beta=\max \left\{\left|b_{i}\right|: 1 \leq i \leq m\right\}$, the nonzero component $x_{j}$ of the feasible solution of any base is satisfied:

$$
\frac{1}{m^{m / 2} \alpha^{m}} \leq x_{j} \leq m^{m / 2} \alpha^{m-1} \beta
$$

The target function is represented as a problem

$$
f(x, y)=\sum_{j=1}^{n} c_{j} x_{j}+M \sum_{i=1}^{m} x_{n+i}
$$

Vector $x=\left(x_{1}, x_{2}, \cdots x_{n}\right)^{T}$ for the original set of variables, $y=\left(y_{n+1}, y_{n+2}, \cdots y_{n+m}\right)^{T}$ is vector of artificial variables. We define $\gamma=\max \left\{\left|c_{j}\right|: 1 \leq j \leq n\right\}$, and then we can prove the lemma 2.

Lemma 2: If $\mathrm{x}$ is a feasible solution for the original problem, $(\mathrm{x}, 0)$ is a feasible solution to the problem

$$
f(x, 0)=\sum_{j=1}^{n} c_{j} x_{j} \leq n m^{m / 2} \alpha^{m-1} \beta \gamma
$$




\section{Evaluation of Parameter $M$ in the Big M Method}

Theorem 1: If there is an optimal solution for the original problem, and $M_{0}=2 \mathrm{~nm}^{m} \alpha^{m-1} \beta$ is set up, then the $\mathrm{M}>\mathrm{M}_{0}$ is the optimal solution of the original problem.

Prove: Let $\left(\mathrm{x}^{*}, \mathrm{y}^{*}\right)$ is a propagation problem a basic optimal solution. First, we prove that the solution of artificial variables is zero, that $\mathrm{y}^{*}$. Otherwise, with an artificial variable $\mathrm{x}^{*} \mathrm{n}+\mathrm{I}>$, by lemma 1

$$
\begin{gathered}
x_{n+i}^{*} \geq \frac{1}{m^{m / 2} \alpha^{m}}, \\
x_{j}^{*} \leq m^{m / 2} \alpha^{m-1} \beta(1 \leq j \leq n),
\end{gathered}
$$

So we have

$$
\begin{gathered}
f\left(x^{*}, y^{*}\right)=\sum_{j=1}^{n} c_{j} x_{j}^{*}+M \sum_{i=1}^{m} x_{n+i}^{*} \\
>-n m^{m / 2} \alpha^{m-1} \beta \gamma+M_{0} \frac{1}{m^{m / 2} \alpha^{m}} \\
=-n m^{m / 2} \alpha^{m-1} \beta \gamma+2 n m^{m / 2} \alpha^{m-1} \beta \gamma \\
=n m^{m / 2} \alpha^{m-1} \beta \gamma
\end{gathered}
$$

On the other hand, since the original design problem exist the optimal solution, there are some feasible solutions, and the feasible solution $\mathrm{X}_{0}$. $\left(\mathrm{X}_{0}, 0\right)$ is the feasible solution of the problem.

$$
f\left(x^{0}, 0\right)=\sum_{j=1}^{n} c_{j} x_{j} \leq n m^{m / 2} \alpha^{m-1} \beta \gamma<f\left(x^{*}, y^{*}\right)
$$

And $\left(x^{*}, y^{*}\right)$ is the optimal solution to the problem of expansion, which is proved by $\mathrm{y}^{*}=0$. By

$y *$ know $\mathrm{x} *$ to the original set of feasible solution. The original design problem for an arbitraryfeasible solution $\mathrm{x}(\mathrm{x}, 0)$ is naturally extended problems feasible, that

$$
\text { is } f\left(x^{*}, 0\right) \leq f(x, 0) . \sum_{j=1}^{n} c_{j} x_{j}^{*} \leq \sum_{j=1}^{n} c_{j} x_{j}
$$

Therefore, $\mathrm{x} *$ is for the original design problems of the optimal solution. Inference when the $m>M 0$. If in the extension of the optimal solution is an artificial variables $X_{n+i}>$, original of will not exist feasible solution. Expansion in solving problems in the process, when all artificial variablesare zero, may still have some artificial variables in the medium. We cannot ignore them, although as usual pivoting operation. Unlike the two-stage method,it must be to expel base all artificial variables, can be transferred to the second stage, so the big $\mathrm{M}$ method can reduce the handover procedure of two stages.

We obtain a constant $M_{0}=2 \mathrm{~nm}^{\mathrm{m} / 2} \alpha^{m-1} \beta \gamma$, which only depends on the original problem of the input data, and proves that when the $\mathrm{M}>\mathrm{M}_{0}$ method is effective, or convergence of the $\mathrm{M}$ method.

So the data $\mathrm{M}_{0}$ input length is less than or equal to

$$
2 \log n+m \log m+(2 m-1) \log \alpha+\log \beta+\log \gamma
$$

It is the original set of linear programming input length polynomial. Therefore, from the point of view of the computational complexity, data of $\mathrm{M}_{0}$ (i.e., the storage space it occupies) size is normal, is from the original problem to extend the conversion problem that meet the definition of polynomial reduction. Of course, further reducing $\mathrm{M}_{0}$ valuation is worthy of study. For all linear 
programming structure coefficients, the $\mathrm{M}_{0}$ is the most conservative estimates. But on the special structure of the coefficient or removed in the feasible solution set is not necessary, the $\mathrm{M}_{0}$ value can be reduced.

\section{References}

[1] Zhang Zhongwen, Wang Shihui, Several Kinds of Special Cases of Finding Optimal Solution for Linear Programming Problem by the Simplex Algorithm, J. Journal of Gansu Lianhe University (Natural Sciences).3(2010) 101 - 103.

[2] Mao Baohua, Li Wei, Hu Xing xing, One Artificial Variable Big “M” Methods with Deficient Basis, J.Journal of Hangzhou Dianzi University(Natural Sciences). 6(2010) 66 - 70.

[4] Wang Jiqiang, An Analysis of the Consistency of Big M Method and Two-Phase Method, J. College Mathematics.12(2004) 106 - 108 